\section{Molecular Roles of GREB1 in ER-Positive Breast Cancer}

\section{Abstract}

Growth regulation by estrogen in breast cancer 1 (GREB1) is one of the top estrogen (E2) responsive estrogen receptor (ER) target gene. GREB1 play pivotal role in the ER signaling dependent oncogenesis of breast cancers. GREB1 has been reported as a regulatory factor in ER signaling as it interact and regulate the function of $E R \alpha$; the predominant subclass of ER. GREB1 acts as transcription coactivator that affects ER-chromatin interaction thereby modulate its downstream oncogenic signals that initiate the development and progression of breast cancer. Such intimate role of GREB1 places it as a therapeutic target and clinical biomarker for patient's response to endocrine therapy. More recently Tamoxifen resistance in breast cancer was found to be regulated by the EZH2-ER $\alpha-G R E B 1$ transcriptional axis. Despite the presence of such discrete evidence on the involvement of GREB1 in triggering the oncogenesis as well as drug response of breast cancer, there is very few compiled reports on the possible molecular mechanism how GREB1 could affect ER-associated tumor growth and subsequent therapeutic responses. Hence this review is written on the molecular roles of GREB1 in ER-positive breast cancer.

Keywords: GREB1; Breast; Cancer; Estrogen receptor alpha; Oncogenesis

\begin{abstract}
Neja SA*
College of Natural and Computational Science, Faculty of Veterinary Medicine, Hawassa University, P.O. Box 05, Hawassa, Ethiopia
\end{abstract}

\section{* Corresponding author:}

Dr. Sultan Abda Neja

” sultanabda@gmail.com

College of Natural and Computational Science, Faculty of Veterinary Medicine, Hawassa University, P.O. Box 05, Hawassa, Ethiopia.

Tel: $+251-940017269$

Citation: Neja SA (2020) Molecular Roles of GREB1 in ER-Positive Breast Cancer. Insights Biomed Vol.5 No.1:2

\section{Introduction}

ER-positive breast cancer is the most common type of breast cancer. It constitutes more than $75 \%$ of breast cancers. GREB1 is highly expressed in ER-positive breast cancers and it is one of the E2-induced ER target genes [1-4]. Knockdown of GREB1 results in inhibition of cell proliferation in several hormonedependent cancers. Recently, GREB1 was reported to function as a transcription co-activator of ER $\alpha$. Loss or dysregulation of GREB1 substantially decreased ER $\alpha$-mediated gene transcription and reduced tumor growth $[1,2,4,5]$. One study found GREB1-ER $\alpha$ interactions in $50 \%$ of ER+ cancers and showed GREB1 expression was correlated with a good clinical outcome [1]. Nevertheless, little is known about the exact role of GREB1 in the cascade of hormone action, though it appears to be a key E2-induced gene having role in ER signaling.

In ER signaling pathway, E2 induces the expression of ER target genes with a subsequent effect on cancer cell growth, survival and metastasis. GREB1 is well known ER target gene reported to be critically involved in the E2-induced breast cancer growth and as a clinical marker for patients' response to endocrine therapy [1]. More recently GREB1 was reported to be involved in modulating ER signaling in which SIGREB1 affect ER-chromatin interaction [5]. Despite the fact that GREB1 is well known as top E2 responsive ER target regulating ER signaling, there is very few reports on the detailed molecular mechanism how GREB1 affect ER-associated tumor growth and subsequent therapeutic responses. This review is thus elucidates the molecular roles of GREB1 in the oncogenesis as well as drug response of ER-positive breast cancer.

\section{Literature Review}

\section{Genomic location of GREB1 and its Isoforms}

GREB1 is encoded on the plus strand of chromosome 2, at 2q25.1, covering $108.67 \mathrm{~kb}$ from 11674242 to 11782912 (Table 1). The gene structure of GREB1 consists of 60 alternative exons and 40 different introns. The partial GREB1 sequence was first cloned from the size-fractioned adult brain cDNA library in 1998 and named KIAA0575 [6]. Two years later, the three primary representatives GREB1 complete cDNA clones were isolated from an ER+ breast cancer cell line (MCF7) CDNA library and named as GREB1a, GREB1b and GREB1c respectively [7]. The three transcript variants contain distinct c-terminus regions on genomic locus; differ in the number of exon and a total base pair (Table 1). More splice variants have been also reported in breast, uterus, prostate and brain $[6,8]$. 
Table 1 Sequence organization of the three isoforms of GREB1.

\begin{tabular}{|l|c|c|c|c|c|c|c|}
\hline Isoforms & Genomic location & Accession & Length (aa) & Molecular mass (KDa) & Exon & Base pair \\
\hline GREB1a & $11674242-11782912$ & NP_055484 & 1949 & 216.5 & 33 & 8482 \\
\hline GREB1b & $11682851-11732446$ & NP_149081 & 457 & 48.9 & 11 & 2521 \\
\hline GREB1C & $11679969-11728353$ & NP_683701 & 409 & 43.2 & 10 & 2432 \\
\hline
\end{tabular}

The GREB1 gene contains three consensuses EREs motif located at $1.5 \mathrm{~kb}, 9.5 \mathrm{~kb}$, and $21 \mathrm{~kb}$ upstream of the transcription start site (TSS) [9]. The ERE found at $1.5 \mathrm{~KB}$ upstream of TSS is considered as a GREB1 core promoter where ER $\alpha$ binds. The ERE found 9.5 $K B$ upstream of TSS and the $21 \mathrm{~kb}$ distal enhancer cooperate for transcriptional induction of the GREB1 gene by ER $\alpha$ through making chromatin loops. In breast cancer cells, the steroid receptor co-activator SRC-3, phosphorylated RNA polymerase II and acetylated histones are also bound to these ER $\alpha$-ERE complexes in the presence of E2 as evidenced by chromatin immunoprecipitation (ChIP) assay $[10,11]$. These findings on the genomic location of GREB1 indicate that GREB1 is an ER $\alpha$ target gene.

\section{Oncogenic roles GREB1 in ER-positive breast cancer}

The oncogenesis of breast cancer has been associated with $E R \alpha$; the predominant subclass of ER in ER signaling pathway. ER $\alpha$ play its oncogenic role by causing deranged activation its target genes. Among the ER target genes, GREB1 have been reported to be involved in the E2-induced breast cancer growth, survival and metastasis. This is evidenced by the presence of positive correlation ERo-positivity of breast cancer cell lines and GREB1 mRNA and protein expression as well as chromatin binding study so far conducted shows GREB1 as a downstream target of ER $\alpha$ $[1,5]$. This pattern also correlates with the progression of various hormone-dependent cancers such as breast, ovary, and prostate cancers [1-4]. On top of this it has been shown that GREB1 knockdown significantly reduces tumor growth $[1,2,4]$.

Mechanistically GREB1 was found to function as a transcription co-activator of $E R \alpha$ by bridging $E R \alpha$ and other transcription activators such as CREB-binding protein/P300 histone acetyltransferases thus it mediate interactions between the ER and additional proteins through which it affects the expression of E2-induced oncogenic ER target genes. In the same study, the removal of GREB1 substantially decreases ER $\alpha$-mediated gene transcription and cell proliferation [5]. Upon GREB1 knockdown in MCF-7 cells, nearly half of the estrogen- responsive genes were no longer differentially expressed, and the cells were less able to form colonies [5]. In further elucidation of the above mechanism compelling reports have shown the presence of GREB1 sensitizes E2-responsive breast cancer to endocrine therapy [12]. Consequently, it indicates that on top of the conventional ERtargeted therapy, GREB1 could be a potential therapeutic target for hormone sensitive ER-positive breast cancer.

In the oncogenesis of breast cancer, E2 influences uncontrolled cell proliferation or promote ER independent signaling to sidestep the physiologically controlled ER signaling $[13,14]$. Few reports also showed that breast cancers express a protein that stabilizes
ER $\alpha$ to promote proliferation $[15,16]$. Since GREB1 is a well characterized E2-responsive gene used to identify $E R \alpha$ activity and silencing GREB1 can affects the ER oncogenic signatures known to regulate the proliferation of breast cancer cells and its phenotypic properties, further detail molecular mechanism how GREB1 play this role or weather GREB1 reported to bind ER $\alpha$ could regulate $E R \alpha$ stability thereby $E R \alpha$ function is remain to be elucidated [5,17-20].

\section{GREB1 modulate tamoxifen response of ER+ breast cancer}

More recently loss of GREB1 has been reported to be involved in the resistance to ER-targeted endocrine therapy. As GREB1 is an ER target gene, persistent ER $\alpha$ targeted tamoxifen treatment could leads to gradual loss of GREB1 in with those initially hormone sensitive ER-positive cells rather start to rely on the alternative mechanism for growth and survival (Figure 1). Beside this, GREB1 has been depicted to mediate interactions between the $E R \alpha$ and other proteins, as an inhibitory factor in tamoxifenliganded ER complex [5].

On top of this, patient's correlation study showed that the expression of GREB1 is epigenetically silenced by enhancer of zeste homolog 2 (EZH2) in Tamoxifen-resistant cases [21]. Yet these findings indicate that maintenance of GREB1 protein level is important to balance the $E R \alpha$ - dependent transcriptional responses. As indicated in Figure 1 right panel, EZH2-medited epigenetic silencing decreases the expression GREB1 thereby trigger Tamoxifen resistance. Indeed, the role of epigenetic regulation in E2-responsiveness has come to light as a potential treatment target to revert hormone therapy-resistant breast cancers [22,23]. However, the detailed mechanism how the presence of GREB1 sensitizes the cells and the reason why GREB1 needs to be downregulated for the acquisition of endocrine resistance remains unclear suggesting the need for further study for the mechanistic role of GREB1 in the pathophysiology of breast cancer.

Nevertheless, ER $\alpha$ is a master regulator and a well-validated drug target for a majority of breast cancers, yet the mechanisms by which agonist-bound ER $\alpha$ causes repression and the mechanism of resistance to such drug action are poorly understood. Although GREB1 is known to bind to ER $\alpha$ and associated effects were also consistent across those studies, the direct effect of GREB1 binding on $E R \alpha$ is yet to be disclosed.

\section{GREB1 as biomarker in the breast cancer}

Tumor ER $\alpha$ status is routinely determined as it predicts for response to hormonal therapy. However, the predictive power of $E R \alpha$ alone is limited and tumor ER $\alpha$ status fails to identify those more likely to recur after hormonal therapy (suggestive of de novo 


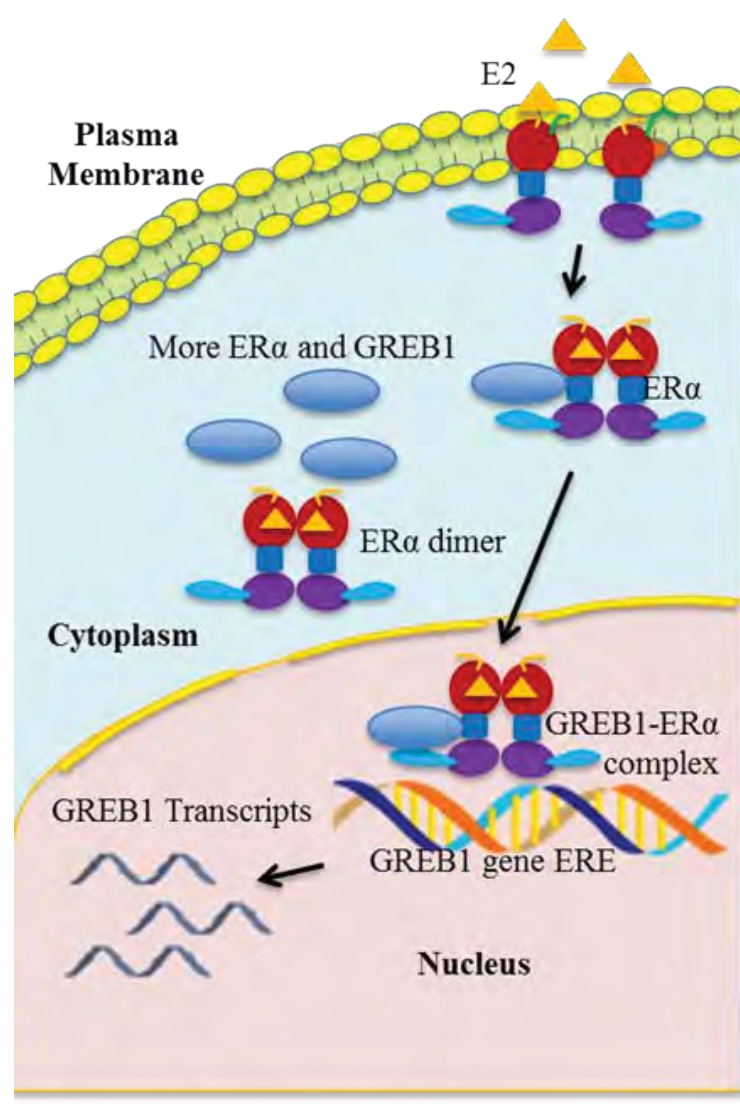

Tamoxifen Sensitive

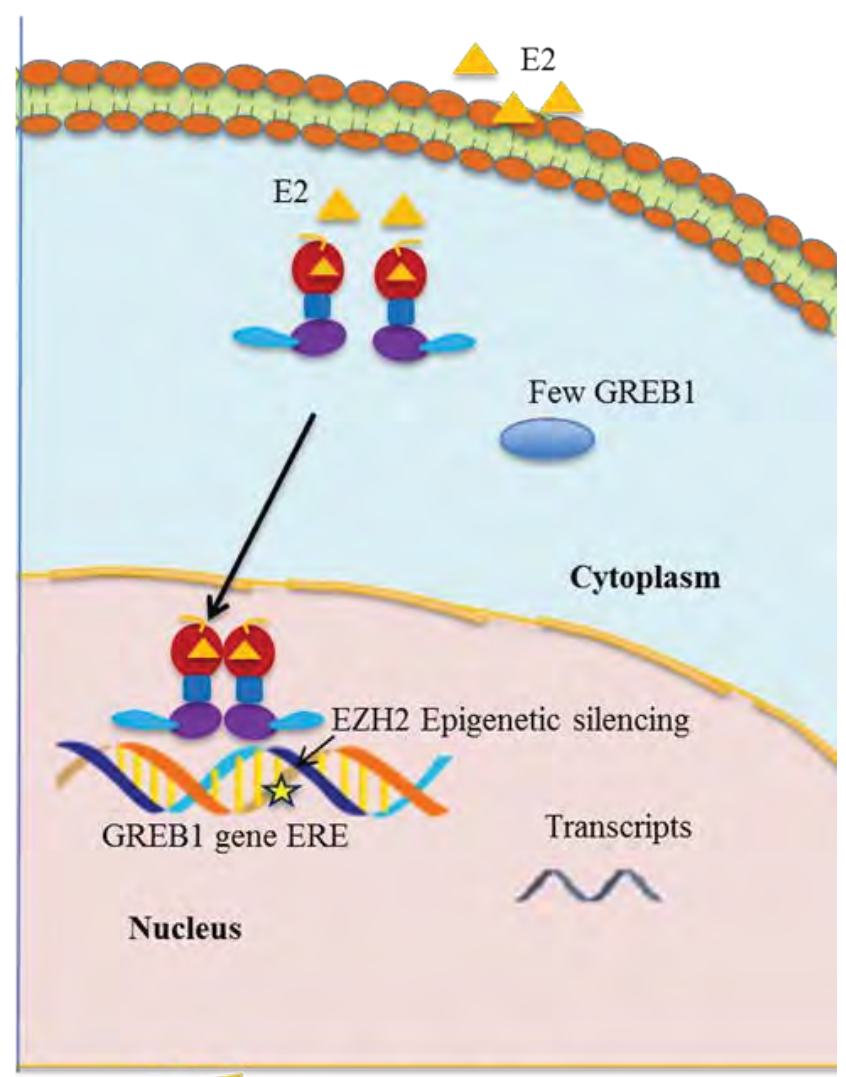

Tamoxifen Resistance

Figure 1 Roles of GREB1 in E2-induced ER signalling pathways of ER-positive cells. GREB1 gene has ERE where ER $\alpha$ binds and induces its expression. Tamoxifen sensitive cells have more GREB1 expression which subsequently interacts with ER $\alpha$ (Left Panel) to further boost its own expression. While in Tamoxifen resistant cells, EZH2-medited epigenetic silencing decreases the expression GREB1 (right panel).

or acquired endocrine resistance) $[24,25]$. This may be related to the way in which ER $\alpha$ positivity is defined. IHC is routinely used for evaluation of $E R \alpha$, and a positive result is defined according to scoring systems that take into account staining intensity and proportion of positively-stained cells. In the case of ER $\alpha$, several scoring systems are used and since the positive threshold for each differs, the definition of ER-positivity is somewhat ambiguous [26-28]. Furthermore, this threshold can sometimes be modified [29]. Low PR expression was an independent predictor of relapse, as was high Ki67 expression [30,31]. As a result, biomarkers like $\mathrm{PR}$, major ER $\alpha$ target genes or downstream effector arms of $\mathrm{ER} \alpha$ activation, and Ki67 (a marker of proliferation) have been evaluated in combination with $E R \alpha$ [32].

Because of the lack of perfect correlation between ER and/or PR status and patient's response to hormone therapy, there was a need to identify additional protein markers that would improve prediction of hormone response. GREB1 has been reported as potential molecular markers not only in breast cancer but also for prostate cancer prognosis and more exceptionally in saliva samples for lung cancer $[5,7,33,34]$. On top of this GREB1 with Paired-box gene, 8 (PAX8) has been depicted as a biomarker in ovarian cancer [34]. Concurrently same groups also depicted GREB1 to underlie E2 induced progression of ovarian tumor and thus may act as a targetable molecule [4]. These all findings illustrate that GREB1 as an effector arm of ER signaling is critical to improve prognostication.

\section{Conclusion}

GREB1 is one of the top E2 responsive ER target gene. It play pivotal role in the ER signaling dependent oncogenesis of breast cancers. Mechanistically GREB1 interact and regulate the function of ER $\alpha$. As transcription coactivator, GREB1 affects chromatin interaction of ER $\alpha$ thereby regulates the expression of oncogenic signatures known to trigger the development and proliferation of breast cancer cells and its phenotypic properties. In this regard GREB1 has been depicted to be used as therapeutic target and clinical biomarker for patient's response to endocrine therapy. On top of this loss of GREB1 has been reported to be involved in the tamoxifen resistance in breast cancer. Such evidence on the involvement of GREB1 in triggering the tumor growth as well as its drug response, suggests the need for detail study on the molecular mechanism how GREB1 could affect $E R \alpha$ function 
and ER-associated oncogenesis and subsequent therapeutic responses of ER-positive breast cancer.

\section{Acknowledgements}

The author acknowledges Hawassa University for providing

\section{References}

1 Rae JM, Johnson MD, Scheys JO, Cordero KE, Larios JM, et al. (2005) GREB 1 is a critical regulator of hormone dependent breast cancer growth. Breast Cancer Res Treat 92: 141-149.

2 Rae JM, Johnson MD, Cordero KE, Scheys JO, Larios JM, et al. (2006) GREB1 is a novel androgen-regulated gene required for prostate cancer growth. Prostate 66: 886-894.

3 Hnatyszyn HJ, Liu M, Hilger A, Herbert L, Fernandez CRG, et al. (2010) Correlation of GREB1 mRNA with protein expression in breast cancer: Validation of a novel GREB1 monoclonal antibody. Breast Cancer Res Treat 122: 371- 380.

4 Laviolette LA (2014) 17beta-estradiol upregulates GREB1 and accelerates ovarian tumor progression in vivo. Int J Cancer 135: 1072-1084.

5 Mohammed H (2013) Endogenous purification reveals GREB1 as a key estrogen receptor regulatory factor. Cell Rep 3: 342-349.

6 Nagase T (1998) Prediction of the coding sequences of unidentified human genes. IX. The complete sequences of 100 new cDNA clones from brain which can code for large proteins in vitro. DNA Res 5: 31-39.

7 Ghosh MG, Thompson DA, Weigel RJ (2000) PDZK1 and GREB1 are estrogen-regulated genes expressed in hormone-responsive breast cancer. Cancer Res 60: 6367-6375.

8 Neto DE (2000) Shotgun sequencing of the human transcriptome with ORF expressed sequence tags. Proc National Acad Sci USA 97 3491-3496.

9 Bourdeau V (2004) Genome-wide identification of high-affinity estrogen response elements in human and mouse. Mol Endocrinol 18: 1411-1427.

10 Sun J, Nawaz Z, Slingerland JM (2007) Long-range activation of GREB1 by estrogen receptor via three distal consensus estrogen-responsive elements in breast cancer cells. Mol Endocrinol 21: 2651-2662.

11 Deschenes J (2007) Regulation of GREB1 transcription by estrogen receptor alpha through a multipartite enhancer spread over $20 \mathrm{~kb}$ of upstream flanking sequences. J Biol Chem 282: 17335-17339.

12 Almodovar A, Zhu X, Litherland S, Decker D (2015) Promestriene Affects GREB1 expression in estrogen sensitive breast cancer cells. J Cancer Ther 6: 767-772.

13 Yager JD, Davidson NE (2006) Estrogen carcinogenesis in breast cancer. N Engl J Med 354: 270-282.

14 Yue W (2010) Effects of estrogen on breast cancer development: Role of estrogen receptor independent mechanisms. Int J Cancer 127: $1748-1757$.

15 Zhu J (2014) The atypical ubiquitin ligase RNF31 stabilizes estrogen receptor alpha and modulates estrogen-stimulated breast cancer cell proliferation. Oncogene 33: 4340-4351.

16 Zhuang T (2017) SHARPIN stabilizes estrogen receptor alpha and internet facility and reading materials used to prepare this manuscript.

\section{Conflicts of Interest}

The authors declare no conflicts of interest.

promotes breast cancer cell proliferation. Oncotarget 8: 7713777151.

17 Cai W (2011) The immunophilin-like protein XAP2 is a negative regulator of estrogen signaling through interaction with estrogen receptor alpha. PLoS One 6: e25201.

18 Gupta N, Grebhardt S, Mayer D (2012) Janus kinase 2--a novel negative regulator of estrogen receptor alpha function. Cell Signal 24: 151-161.

19 Liu M (2012) GREB1 functions as a growth promoter and is modulated by IL6/STAT3 in breast cancer. PLoS One 7: e46410.

20 Wu Y (2017) Tamoxifen resistance in breast cancer is regulated by the EZH2-ERalpha-GREB1 transcriptional axis. Cancer Res 2: 2-4.

21 Meng Q (2011) Carbamazepine promotes Her-2 protein degradation in breast cancer cells by modulating HDAC6 activity and acetylation of Hsp90. Mol Cellul Biochem 348: 165-171.

22 Yardley DA (2013) Randomized phase II, double-blind, placebocontrolled study of exemestane with or without entinostat in postmenopausal women with locally recurrent or metastatic estrogen receptor-positive breast cancer progressing on treatment with a nonsteroidal aromatase inhibitor. J Clin Oncol 31: 2128-2135.

23 Miller TW (2013) Endocrine resistance: What do we know? Am Soc Clin Oncol Educ Book.

24 Jankowitz RC (2017) New strategies in metastatic hormone receptorpositive breast cancer: Searching for biomarkers to tailor endocrine and other targeted therapies. Clin Cancer Res 23: 1126-1131.

25 Qureshi A, Pervez S (2010) Allred scoring for ER reporting and its impact in clearly distinguishing ER negative from ER positive breast cancers. J Pak Med Assoc 60: 350-353.

$26 \mathrm{Ma} \mathrm{H}$ (2013) Quantitative measures of estrogen receptor expression in relation to breast cancer-specific mortality risk among white women and black women. Breast Cancer Res 15: R90.

27 Mudduwa LK (2009) Quick score of hormone receptor status of breast carcinoma: Correlation with the other clinico-pathological prognostic parameters. Indian J Pathol Microbiol 52: 159-163.

28 Hammond ME (2010) American Society of Clinical Oncology/College of American Pathologists guideline recommendations for immunohistochemical testing of estrogen and progesterone receptors in breast cancer. J Clin Oncol 28: 2784-2795.

29 Nishimukai A (2015) High Ki-67 expression and low progesterone receptor expression could independently lead to a worse prognosis for postmenopausal patients with estrogen receptor- positive and HER2-negative breast cancer. Clin Breast Cancer 15: 204-211.

30 Purdie CA (2014) Progesterone receptor expression is an independent prognostic variable in early breast cancer: A population-based study. Br J Cancer 110: 565-572.

31 Daniel AR, Hagan CR, Lange CA (2011) Progesterone receptor action: Defining a role in breast cancer. Expert Rev Endocrinol Metab 6: 359-369. 
32 Antunes AA (2012) GREB1 tissue expression is associated with organconfined prostate cancer. Urologic Oncol 30: 16-20.

33 Zhang L (2012) Development of transcriptomic biomarker signature in human saliva to detect lung cancer. Cellular Mol Life Sci 69: 3341-3350.
34 Hodgkinson K (2016) The role of steroid hormones, GREB1, and reproductive status in ovarian cancer progression, in Department of Cellular and Molecular Medicine. University of Ottawa, Ontario, Canada. 